\title{
Public Health Nursing Acceptance of the 5 A's Protocol for Prenatal Smoking Cessation
}

\author{
Suzanne H. Yusem, Kenneth D. Rosenberg, Lesa Dixon-Gray, Jihong Liu \\ Oregon Department of Human Services, Office of Family Health
}

\begin{abstract}
Oregon's efforts in tobacco cessation have historically focused on the general population and have depended on quit line services as the primary intervention. The Oregon Smoke Free Mothers and Babies Program (SFMB) was developed in 2002 to focus on public health nurses and prenatal care providers who work with high risk pregnant women. It seeks to increase smoking cessation among low income and other high risk pregnant women by disseminating the U.S. Public Health Service best practices, the 5 A's (Ask, Advise, Assess, Assist, Arrange) tobacco brief intervention protocol, to public health nurses and prenatal care providers. Interventions included teaching nurses the $5 \mathrm{~A}$ 's, how to use stages of change for pregnant quitters and providing them with client materials. We report the survey results gathered from nurses regarding their use of the 5 A's. Nurses were questioned at 3 intervals: at the beginning of the SFMB project, 12 months later and 24 months later. While over 45 nurses in 10 counties were involved in the program, staff turnover and budget cuts affected program evaluation and analysis of the survey responses. As a result, only 10 nurses completed all three surveys. We found that, at baseline, all of the nurses were already performing the Ask and Advise components. The training resulted in a significant increase in the nurses using Assess $(\mathrm{p}<0.05)$ and Assist $(\mathrm{p}<0.05)$ both at 12 and 24 months. We also found that there was a statistically significant increase in the use of Arrange at 12 months $(p<0.01)$ that was not sustained at 24 months $(\mathrm{p}=0.07)$. We conclude that public health nurses were already routinely doing Ask and Advise; our 5 A's program was successful in improving Assess and Assist. More work is needed to understand why increases in Arrange were not sustained.
\end{abstract}

(C) 2004 Californian Journal of Health Promotion. All rights reserved.

Keywords: tobacco, prenatal care, smoking cessation, Oregon

\section{Introduction}

In Oregon, tobacco cessation activities have typically occurred through the Oregon Tobacco Quit Line focusing on a broad population base. The Quit Line was established in 1998 for all Oregonians who smoked and wanted to quit. The Quit Line provides free telephone counseling to all callers, but they do no outreach: callers must contact them for services. Specific cessation interventions, while recommended, were not routinely being conducted by providers in public health or private offices. The Oregon Office of Family Health administers a home-visiting program called Maternity Case Management (MCM) for high-risk pregnant women. The program is funded through the US Title V program and the
Oregon State Medicaid Program. State rules for the MCM program required that tobacco use be assessed for every client. Those clients who smoked were considered "high-risk" and could receive additional services. However, specific methods for assessing tobacco use were not spelled out for nurses. Nurses had received little information and training regarding tobacco cessation or motivational interviewing. They were also unfamiliar with cessation resources.

\section{Oregon Smoke Free Mothers and Babies (SFMB)}

In an effort to focus more directly on the population of pregnant women in Oregon and address the need for nurses and providers to screen and assist women to quit smoking, the 
Smoke Free Mothers and Babies Program (SFMB) was developed. The goal of the program, funded by the National Dissemination office of Smoke Free Families (at the University of North Carolina at Chapel Hill, funded by the Robert Wood Johnson Foundation), was to increase smoking cessation among low income and other high risk pregnant women through introducing the $5 \mathrm{~A}$ 's brief intervention protocol to prenatal care providers and public health nurses. This paper addresses the portion of the program that taught public health nurses how to help pregnant women stop smoking. Activities included training nurses and providing them with client materials and streamlining existing systems that allowed nurses to use the 5 A's with greater ease.

Ten counties in Oregon were originally recruited for the project. They were culled from a total of 36 counties in Oregon, nine of which were not eligible because of their involvement in another Smoke Free Families Project or their lack of a Maternity Case Management (MCM) program. Out of the remaining 27 counties, 10 agreed to participate. During the course of the project, two counties discontinued their participation.

In agreeing to participate in the program, County Health Department Nurses providing MCM services were required to recruit pregnant women who smoked into the project and to provide additional documentation, including a project consent form, the client's reports on smoking cessation activities by their provider during prenatal visits, and a client postpartum survey. Nurses were required to complete three surveys during the course of the project. Finally, the "Five A's Intervention Record" (FAIR) Form was introduced, which required public health nurses to document use of the cessation interventions by checking boxes, rather than through written progress notes.

\section{The 5 A's}

The U.S. Public Health Service's Clinical Practice Guidelines (Fiore, et al., 2000) recommend the use of the 5 A's as a brief clinical intervention for health care providers. After reviewing the results from randomized clinical trials involving the 5 A's brief intervention, Melvin et al. (2000) recommended the 5 A's for use in working with pregnant women who smoke. The estimated that total contact time for this procedure ranges from 5 to15 minutes, plus additional time needed to read self-help materials. Specifically, they identified the 5 A's for pregnant women as:

ASK the patient about her smoking status, ADVISE to quit smoking with personalized messages for pregnant women,

ASSESS her willingness to quit in next 30 days,

ASSIST with self-help materials and social support, and

ARRANGE to follow-up during subsequent visits.

A meta-analysis of clinical trials indicated that when the 5 A's brief counseling intervention is used by a trained provider, and is accompanied by pregnancy-specific, self-help materials, cessation rates can be increased by $30-70 \%$ (Mullen, 1999). However, a survey showed that only $35 \%$ of providers used the full 5 A's intervention, with most providers only asked and advised about smoking (Floyd et al., 2001). This is consistent with Oregon data that indicates that $60 \%$ of prenatal care providers used three of the recommended 5 A's protocol (Ask, Advise, and Assist) (Oregon Pregnancy Risk Assessment Monitoring System, 2001). (We have no data on Assess and Arrange.) Other studies have also addressed the lack of consistency with which providers identify smoking status, advise cessation, and provide counseling to their patients who smoke (Thorndike, 1998).

Smoke Free Mothers and Babies Program was designed using DiClemente and Prochaska's Stages of Change model (1998) and Rogers' Diffusion of Innovations Theory (2004) to disseminate the $5 \mathrm{~A}$ 's brief intervention and motivational interviewing. The $5 \mathrm{~A}$ 's is seen as the vehicle to disseminate change in perinatal systems, both Maternity Case Management and private Prenatal Care Providers. An important component used with the "Assist" piece of the 5 A's is Rollnick's Motivational Interviewing (1995). Motivational interviewing is a counseling strategy used to encourage, or 
motivate, behavior change (Miller, 1999). Motivational interviewing is often paired with the Stages of Change model.

Diffusion Theory is based on spreading an idea or innovation through both formal and informal communication channels. With the 5 A's as the innovation, SFMB was charged with getting nurses excited about the changes. According to Rogers' Theory, once 15 percent of a group adopts a new theory, others in the group will follow. SFMB planned on diffusing tobacco cessation best practices through Oregon's Maternity Case Management System and then through the prenatal care providers.

Prior to this project, the most widely used and available cessation intervention for all Oregonians has been the Oregon Tobacco Quit Line (http://www.oregonquitline.org). Pregnant women received quit line services specifically tailored to issues around pregnancy. Services included a twenty to forty minute phone call, "Quit Kit" materials, information on local cessation programs that their insurance carrier would cover, and a later call-back. The Quit Line provided reactive services; the women who needed services had to initiate the contact.

Part of the SFMB program design was to include the Quit Line as a resource for nurses when conducting the "Assist" piece of the intervention. One variation in the standard quit line services was made. Instead of a reactive process, the quit line would be proactive, calling women who had been referred. Several organizations at that time were evaluating a fax referral process to the quit line. SFMB decided to incorporate that process and encourage public health nurses and prenatal care providers to fax their referrals, rather than simply giving the smoking client a phone number. In addition, nurses would fax client tobacco use information (including information about quit line referrals) to the client's prenatal care provider. Thus, the three-pronged approach to SFMB was developed including nurses, the prenatal care providers, and the Oregon Tobacco Quit Line.

\section{The Oregon Program}

Since January 2002, Smoke Free Mothers and Babies has been implementing a process to disseminate the 5 A's to nurses in county Public Health Departments and private prenatal care providers in eight counties throughout Oregon. A three-pronged system -- built on the concept of collaboration between public health nurses, prenatal care providers and the Oregon Quit Line -- has been developed to increase providers' use of the 5 A's. The SFMB's coordinator trained public health nurses and prenatal care providers on how to use the $5 \mathrm{~A}$ 's protocol and to provide motivational counseling. They were also trained to refer women to the Oregon Quit Line through a fax referral process. In some cases, the newly trained nurses were asked to train other public health nurses. Brochures, posters, and other materials were provided on a continuing basis throughout the program to assist women in quitting and to remind providers about cessation practices. Other strategies to incorporate the $5 \mathrm{~A}$ 's into the clinical practice nursing systems included assistance in establishing a documentation system, incorporating the $5 \mathrm{~A}$ 's screening into other prevention systems (i.e., SIDS and asthma), and feedback about tobacco use in their counties.

While the SFMB Program began with the initial training, other interventions that addressed clinic systems were implemented throughout the program. The concept was to introduce the nurses to the 5 A's and motivational interviewing in the initial training and follow them up over the course of the 2 years so that nurses could easily integrate the information into their practice. Thus, after the training, we began a regular practice of sending all participating counties materials for nurses to use. There were one-time mailings of brochures specifically for providers marketing continuing medical education credits for free web-based tobacco training. Towards the end of the two years, a second-hand smoke campaign was implemented. Other materials were routinely sent to nurses over the program period. Specific booklets and tear-off sheets for clients were used by the nurses in copious amounts. Those client 
materials were provided by our funder, Smoke Free Families - National Dissemination Office.

Nurses participated in the development of the documentation form, the Five A's Intervention Record (FAIR) Form that was designed to be used when they administered the 5 A's. This form was used to collect data for the project and served the purpose of a reminder system for the nurses to conduct the brief intervention. Also it was used as a documentation tool for nurses to keep track of their clients' smoking status at each visit. Because they were required to use the form at every visit and with every pregnant woman who smoked, nurses were prompted to document their use of the brief intervention.

Tobacco cessation issues were also addressed in nursing interventions with patients who had other prenatal concerns. Nurses were provided information and materials throughout the program on the effects of tobacco use on Sudden Infant Death Syndrome and Asthma. They received clinical information in the form of articles and research on how tobacco affects these conditions as well as other chronic illnesses. In effect, the program at the State level involved motivating nurses to become invested in addressing cessation as a natural inclusion in their practice.

To ensure that they remained motivated to address tobacco use on a continual basis, the public health nurses in the counties were provided with data on the demographics of the client population in their counties. As a part of the State system, SFMB had access to birth certificate data and data from clients who had been seen in the system. We were able to provide the counties with a count of pregnant smokers and gave them an idea of whether they were serving the most high-risk population. From birth certificate data, we could give them an estimate of the percentage of pregnant smokers in their community. Other data specifically provided numbers of pregnant smokers being seen. Finally, once we had collected enough data, we were able to give them specifics on how many clients they had impacted with their intervention. We also subtly encouraged a level of competition between the counties, as we provided this feedback to them in a group setting.

Clients eligible for this intervention were pregnant women who had been identified by their prenatal care provider, family planning, or WIC program as being eligible for maternity nurse home visiting services (maternity case management, MCM). After enrollment into MCM those women who were smoking or had quit smoking within the last six months were enrolled into the Smoke Free Mothers and Babies Program. Women seeking MCM services were mostly non-Hispanic white, ages 20-25, single, with less than 12 years of education, and on Medicaid. MCM services include one home assessment visit (to assess safety in the home, nutrition consultation and referral to other services such as WIC) plus up to 10 visits (mostly at the client's home). These home visits are in addition to the client's regular prenatal visits at her prenatal care provider's office.

Public Health nurses providing MCM services were trained in the $5 \mathrm{~A}$ 's brief intervention and motivational interviewing. Throughout the training, nurses were asked to conduct the 5 A's every time they had contact with their smoking pregnant clients. They were instructed in methods to assess their client's stage of change and readiness to quit. All nurses in the project were expected to conduct the "Arrange" component of the 5 A's by making future plans with their client to follow up on their smoking status. They were also provided with educational materials for clients and Oregon Quit Line Fax Referral Forms to which they could easily refer their clients.

\section{Research Methods Data Collection}

A longitudinal pretest-posttest study design was used to evaluate how nurses incorporated the 5 A's brief intervention into their daily delivery of MCM services. This analysis is based on the data from three surveys of public health nurses working at 8 project counties at three time points: at baseline (just before their initial training, January-February 2002), at 12 months after their initial training (February-March 2003) and at 24 months after their initial training 
(February-March 2004) (Table 1). The number of nurses who responded to our surveys is 21 at baseline, 29 at 12 months after baseline and 26 at 24 months after baseline. However, only 11 nurses responded to both the baseline and 12month surveys; 10 nurses responded to both the baseline and 24-month surveys and 10 nurses responded to all 3 surveys. This was caused by staff turnover of nurses, the discontinuation of the project in two counties, and the participation of newly-hired nurses during the project period. This evaluation will focus on the effects of the intervention on those 10 nurses.

Table 1

Number of public health nurses who completed evaluation surveys.

\begin{tabular}{|l|c|c|}
\hline \multicolumn{1}{|c|}{ Surveys } & Number of respondents & Dates when the survey was administered \\
\hline Baseline & 21 & January-February 2002 \\
\hline 12 months after baseline & 39 & February-March 2003 \\
\hline 24 months after baseline & 26 & February-March 2004 \\
\hline
\end{tabular}

Survey questions focused on nurses' knowledge, attitude, abilities, and behaviors regarding tobacco cessation. Questions ranged from specifics about the 5 A's to systems level issues such as clinic practice and documentation. The baseline survey assessed the nurses' knowledge of the 5 A's, their current use of the 5 A's during prenatal and postpartum visits, how patients' tobacco use was documented, what resources were available at their office to support their patient's quit attempts, barriers that nurses encountered when assessing tobacco use and assisting patients in tobacco cessation, their beliefs about the effects of perinatal smoking, their perception of clients' receptiveness to smoking cessation, their confidence level in providing effective smoking cessation counseling to their clients, and their frequency of contact with clients' prenatal care providers. Most of the questions asked in the baseline survey were also asked in the two follow-up surveys.

\section{Data Analysis}

The outcomes of this analysis are the use of 5 A's brief intervention by the public health nurses before and after the interventions. At each survey, the following questions were used to measure the frequency of using the 5 A's: When a pregnant woman enters case management, 1) how often is she asked about her smoking status; 2) how often is she advised to quit smoking; 3 ) how often is her willingness to quit smoking in the next 30 days assessed; 4) how often is a problem-solving approach used to counsel her ("assist"); and 5) how often does a pregnant smoker who is willing to quit have a follow-up contact arranged? The choices for these five questions were Never, Rarely, Sometimes, Usually, and Always.

To avoid potential unknown bias that might be introduced by the nurses with incomplete survey information, we restricted this analysis to the data from the 10 nurses who responded to all three surveys. The two-tailed Wilcoxon signedrank test was used to assess the differences in using 5'As across time periods (i.e., baseline vs. 12 months, baseline vs. 24 months). Data were analyzed using SPSS 11.0. Probability values of $<0.05$ were considered statistically significant.

\section{Results}

Tables $2 \mathrm{a}$ and $2 \mathrm{~b}$ present the distributions of the 10 nurses' answers to the questions related to their use of the 5 A's in their practice. For the use of Ask component, there was no significant difference in use of the Ask component from baseline to 12 months after the training and from baseline to 24 months after the training. Similarly, no significant change in using the Advise component at 12 months and 24 months after the intervention was observed. 
For the Assess component, in comparison to the baseline, these changes were both significant at 12 months $(\mathrm{p}=.01)$ and 24 months $(\mathrm{p}=.016)$. For the Assist component, there were significant changes at 12 months $(\mathrm{p}=.015)$ and at 24 months $(p=.046)$ after the intervention.
Compared to baseline, the use of the Arrange component at 12 months was improved. The changes from baseline were significant at 12 months after the training $(p=.009)$ and were marginally significant at 24 months after the training $(\mathrm{p}=0.07)$.

Table 2a

The 5 A's use among public health nurses of the SFMB project $(\mathrm{n}=10)$. $^{*}$

\begin{tabular}{|c|c|c|c|c|c|c|c|c|c|}
\hline & \multicolumn{3}{|c|}{ ASK } & \multicolumn{3}{|c|}{ ADVISE } & \multicolumn{3}{|c|}{ ASSESS } \\
\hline & Baseline & $\begin{array}{c}12 \mathrm{mo} . \\
(p=.317)\end{array}$ & $\begin{array}{c}24 \text { mo. } \\
(p=.317)\end{array}$ & Baseline & $\begin{array}{c}12 \mathrm{mo} . \\
(p=.655)\end{array}$ & $\begin{array}{c}24 \text { mo. } \\
(p=.564)\end{array}$ & Baseline & $\begin{array}{c}12 \mathrm{mo} . \\
(p=.010)\end{array}$ & $\begin{array}{c}24 \mathrm{mo} . \\
(p=.016)\end{array}$ \\
\hline Never & 0 & 0 & 0 & 0 & 0 & 0 & 0 & 0 & 0 \\
\hline Rarely & 0 & 0 & 0 & 0 & 0 & 0 & 2 & 0 & 0 \\
\hline Sometimes & 0 & 0 & 0 & 0 & 0 & 0 & 4 & 0 & 0 \\
\hline Usually & 3 & 1 & 1 & 2 & 3 & 3 & 4 & 6 & 7 \\
\hline Always & 7 & 9 & 9 & 8 & 7 & 7 & 0 & 4 & 3 \\
\hline
\end{tabular}

*p-value from the 2-tailed Wilcoxon signed-rank test which compared the differences in use of the 5 A's between baseline, 12 months after the start of the training and 24 months after the start of the training.

Table $2 b$

The 5 A's use among public health nurses of the SFMB project $(\mathrm{n}=10)$ *

\begin{tabular}{|l|c|c|c|c|c|c|}
\hline \multirow{2}{*}{$\begin{array}{c}\text { Frequency } \\
\text { of use }\end{array}$} & \multicolumn{3}{|c|}{ ASSIST } & \multicolumn{3}{c|}{ ARRANGE } \\
\cline { 2 - 7 } & Baseline & $\begin{array}{c}\mathbf{1 2} \text { mo. } \\
(\mathbf{p = . 0 1 4 )}\end{array}$ & $\begin{array}{c}\mathbf{2 4} \text { mo. } \\
(\mathbf{p = . 0 4 6 )}\end{array}$ & Baseline & $\begin{array}{c}\mathbf{1 2} \text { mo. } \\
(\mathbf{p = . 0 0 9 )}\end{array}$ & $\begin{array}{c}\mathbf{2 4} \text { mo. } \\
(\mathbf{p = . 0 7 0 )}\end{array}$ \\
\hline Never & 0 & 0 & 0 & 1 & 0 & 0 \\
\hline Rarely & 2 & 0 & 0 & 3 & 0 & 0 \\
\hline Sometimes & 4 & 1 & 0 & 1 & 1 & 1 \\
\hline Usually & 3 & 4 & 9 & 4 & 4 & 6 \\
\hline Always & 1 & 5 & 1 & 1 & 5 & 3 \\
\hline
\end{tabular}

*p-value from the 2-tailed Wilcoxon signed-rank test which compared the differences in use of the 5 A's between baseline, 12 months after the start of the training and 24 months after the start of the training.

\section{Discussion}

We found that nurses were already doing the Ask and Advise steps of the 5 A's intervention before our training. Their use of the Assess, Assist and Arrange steps increased after the program interventions but the Arrange increases were not sustained through the second year after those interventions.
The challenge in teaching the 5 A's brief intervention comes in getting health care providers to use all of the 5 A's consistently. In assessing nursing behaviors, our baseline data was consistent with other work showing that providers consistently ask patients about their tobacco use and advise them to quit (Oregon Pregnancy Risk Assessment Monitoring System, 2001). And before the SFMB project, public 
health nurses were not consistently using the last and most difficult of the "A's," Assess, Assist, and Arrange. Nurses began using the entire intervention over the course of the project period, with most of behavior changes occurring during year one and maintained throughout the second year.

Assess improvements make sense because the nurses were trained to constantly reassess clients' readiness to quit. The Assess component is usually where providers begin to decrease their persistence in following the best practice intervention (Floyd et al., 2001). The Clinical Practice Guidelines describe Assess as determining the patient's willingness to quit smoking within the next 30 days (Fiore, et. al., 2000). The Assess piece of the 5 A's involves staging the patient according to the Stages of Change. Once the patient's stage of change has been determined, it then becomes easier for the provider to design motivational strategies to move the patient towards quitting. In the beginning of the SFMB program, nurses did not know how to determine patients' willingness to quit and they were not aware of the staging concepts.

We are encouraged that nurses Assist activities remained significant 24 months after the training began, despite unanticipated barriers. The Assist component of the $5 \mathrm{~A}$ 's is time-consuming and requires skill and persistence from providers. In SFMB, we provided many different reminders to nurses that might help their clients formulate a quit plan. We provided materials for nurses to give to their clients. While referral to the Oregon Tobacco Quit Line was included in the program as a resource, state funding for quit line services was cut a year after the project began. Thus referrals for cessation services did not occur as often as was initially planned. Nurses were placed in the position of having to provide motivation, counseling, and problem-solving approaches on their own. Thus, while trained in motivational counseling, nurses were required to implement their training and use the motivational counseling approach as their main intervention for Assist.
The Arrange component involves scheduling follow-up contacts with the client, either in person or via the telephone (Fiore et al., 2000). This was the most difficult component for the nurses: significant increase at 12 months deteriorated somewhat in the second year. We believe that the deterioration was due to vagueness in the Arrange concept. For providers, both Assess and Assist require concrete skills and activities that define those components. The concept of Arrange was more amorphous for the nurses. Because we insisted that the 5 A's be conducted at every visit, nurses were not always sure when they were Arranging at the visit versus when they were back at the beginning of the cycle, Asking, Advising, and Assessing. We asked nurses to document when they informed their clients that they would follow up on their next visit. This was used as an indicator on the Five A's Intervention Record (FAIR). However, the nurses were less likely to document this component.

Getting the nurses to use the 5 A's involved more than the single training conducted at the beginning of the program. Our challenge was to provide them with materials, reminders, and feedback over the course of the program so that we could continually reinforce the brief initial intervention. Nurses received email, "snail" mail, and telephone reminders. Materials sent to them were targeted both to them and to clients. For example, they were sent posters with the 5 A's materials such as pregnancy wheels with 5 A's reminders, and newsletters to reinforce their motivational interviewing skill. They also received, on a regular basis, booklets and tearoff sheets for their clients. Nurses were required to meet quarterly to report on successes and challenges. During these meetings often focused on challenges in implementing the 5 A's, especially Assess, Assist, and Arrange.

Many of the strategies we incorporated were responses to the inevitable challenges that occur in implementing a project. For example, soon after the initial training, we realized that nurses had difficulty recruiting clients into the project. Problem-solving approaches were done with the counties and scripts for recruiting clients were developed. In addition, nurses had no tracking 
system in place to identify which of their clients were participating in SFMB. Through our own data systems, we also realized that nurses weren't always aware of which clients were smoking. Efforts on our part were made to ensure that nurses had easy mechanisms to track which clients needed services. Also, one of the sites serving a fairly transient population was not able to see participating clients more than once, making it difficult to collect data on the consistent delivery of the 5 A's over time. Because of these and other challenges, tracking systems, recruitment systems, paperwork processes, and other clinical office systems were developed locally to allow easier integration of the 5 A's best practice into the entire nursing case management system. The SFMB sites developed their own system for recruiting, tracking and reporting documentation. Some sites developed a spreadsheet to track their SFMB participants and the documentation being sent to the leadership team; others just had a binder where they kept a list of enrolled clients and their documentation.

Another challenge was staff turnover. Over the course of the project, some nurses were laid off, rotated both in and out of the project, and new nurses hired. While this forced the program to address retraining, it also allowed for an opportunity to provide new information to all participants. This challenge incorporated unwanted bias into the survey responses, as newly trained public health nurses who started delivering the 5 A's later in the course of the project were not as familiar as those who started earlier and were trained by the project coordinator. We also note that this turnover resulted in only 10 numbers of nurses who answered all three surveys. Despite these barriers, our results indicate that our 5 A's program was successful in improving the use of Assess and Assist both at 12 months and 24 months after the start of the project as well as in increasing the use of Arrange in the first year of the project.

Nurses became more familiar with the 5 A's intervention over time due to systems changes in addition to training and providing client materials. They were responsible for documenting components of the intervention, including recording the number of cigarettes smoked and planned and actual quit dates. This documentation form (the FAIR form) was required to be completed at every visit. It was seen as confirmation that the intervention was conducted. Because addressing tobacco use by pregnant women is a requirement in the Maternity Case Management Program, filling out this form was seen as an easier alternative to writing a narrative regarding tobacco. This activity alone reinforced memorization and use of the 5 A's by the public health nurses. Subsequently, the FAIR form has been adopted for use statewide. Policy changes have been made to require the $5 \mathrm{~A}$ 's intervention be used for all pregnant women who smoke and receive public health nursing services.

\section{Public Health Education Implications}

Incorporating tobacco cessation best practice interventions into public health nursing practice involves more than just delivery of training and materials. While training can be the beginning of creating a systems change, greater and continuous support is necessary for that change to be sustained over time.

By integrating the 5 A's into everyday nursing practice (such as data collection and documentation, communication reminders, and organizational support) along with consistent training, providing materials, and persistent reminders, public health nurses can consistently conduct tobacco cessation best practices with their patients, as shown in the results reported here.

Health educators routinely encounter barriers when they want to encourage behavior change among health care professionals. Often the answer lies in establishing credibility among respected professionals who agree with the importance of using best practices in a consistent manner. We were fortunate to have the support of state nurses and in working with the local public health nurses. In some cases, it may be easier for health educators to identify a nurse who can be their partner and champion. 


\title{
References
}

DiClemente, C. C., and Prochaska, J. O. (1998). Toward a comprehensive transtheoretical model of change. In Miller, W. R., \& Healther, N. (Eds.), Treating addictive behaviors. (pp. 3-24). New York: Plenum Press.

Fiore, M. C., Bailey, W. C., Cohen, S. J., Dorfman, S. F., Goldstein, M. G., Gritz, E. R., Heyman, R., B. et al. (June 2000). Treating tobacco use and dependence. Clinical Practice Guideline, U.S. Department of Health and Human Services, Public Health Service, Rockville, MD.

Floyd, R. L., Belodoff, B., Sidhu, J., Schulkin, J., Ebrahim, S. H., and Sokol, R. J. (2001). A survey of obstetrician-gynecologists on their patients' use of tobacco and other drugs during pregnancy. Prenatal and Neonatal Medicine, 6, 201-207.

Melvin, C. L., Dolan-Mullen, P., Windsor, R. A., Whiteside, H. P., and Goldenberg, R. L. (2000). Recommended cessation counselling for pregnant women who smoke: a review of the evidence, Tobacco Control, 9 (Suppl 3), III80-4.

Miller, W. R. (1999). Enhancing motivation for change in substance abuse treatment. Treatment Improvement Protocol (TIP), Series 35. Substance Abuse and Mental Health Services Administration, Center for Substance Abuse Treatment.

Mullen, P. D. (1999). Maternal smoking during pregnancy and evidence-based intervention to promote cessation. Primary Care, 26, 577-89.

Oregon Department of Human Services (2001). Oregon pregnancy risk assessment monitoring system. Retrieved October 31, 2004, from http://www.dhs.state.or.us/publichealth/pch/prams/2001/quitsmok.cfm

Rogers, E. M. (1995). Diffusion of innovations. New York: Free Press.

Rollnick, S., and Miller, W. R. (1995). What is motivational interviewing? Behavioural and Cognitive Psychotherapy, 23, 325-334.

Thorndike, A. N.; Rigotti, N.; Stafford, R. S.; Singer, D. E. (1998). National patterns in the treatment of smokers by physicians. Journal of the American Medical Association, 29, 604-608.

\section{Acknowledgements}

The authors would like to thank the nurses at the eight county health departments whose generous cooperation and hard work made this project possible. They would like to thank Pat Westling for conceiving this idea and developing the SFMB program as well as providing support and leadership. SFMB was funded by the National Dissemination Office (SFF-NDO) of Smoke Free Families. Cathy Melvin, Ph.D., MPH and Catherine Rohweder, Dr.PH., MPH of SFF-NDO have provided guidance and consultation throughout the life of this project. We thank them for their support of this work.

\author{
Author Information \\ Suzanne H. Yusem, MS \\ Oregon Department of Human Services \\ Office of Family Health \\ 800 NE Oregon Street, Suite 850 \\ Portland, OR 97232 \\ Ph.: 503-731-4021 \\ Fax.: 503-731-4091 \\ E-mail: suzanne.h.yusem@state.or.us \\ Kenneth D. Rosenberg, MD, MPH \\ Oregon Department of Human Services \\ Office of Family Health \\ 800 NE Oregon Street, Suite 850
}


Portland, OR 97232

Ph.: 503-731-4507

Fax.: 503-731-4091

E-mail: ken.d.rosenberg@state.or.us

Lesa Dixon-Gray, MPH MSW

Oregon Department of Human Services

Office of Family Health

800 NE Oregon Street, Suite 825

Portland, OR 97232

Ph. 503-731-8606

Fax.: 503-731-4091

E-mail: lesa.dixon.gray@state.or.us

Jihong Liu, DSc

Oregon Department of Human Services Office of Family Health

800 NE Oregon Street, Suite 850

Portland, OR 97232

Ph.: 503-731-4021

Fax.: -503-731-4091

E-mail: jihong.liu@state.or.us

* corresponding author 